\title{
Review of: "ROPIUS0: A deep learning-based protocol for protein structure prediction and model selection and its performance in CASP14"
}

jianquan ouyang ${ }^{1}$

1 Xiangtan University

Potential competing interests: The author(s) declared that no potential competing interests exist.

1.ROPIUS0 ranked the tailend with 37.341 GDT-TS in the "Estimate of Model Accuracy" in CASP14. From the description in this article, ROPIUSO and ROPIUSOQA use the same model selection method. Notely, the evaluation score of the paper is different from the computing model of GDT-TS . Even so, it is doubtful that the model can be selected from the prediction structure in the server group and it ranked the final 13th place.

2. And the specific scope of the so-called "independently generated models" is not accurately stated in the paper. For example, taking account to the the previous years' results, such as Zhang, Rosetta, Raptor-X, the performance of them was very good. If it only selected the structure from the better-performing server in previous years for submission, even random selection can achieve better results.

3. If it obtained better Model accuracy estimation by the proposed protocol and the actual GDT-TS Pearson correlation is not high, it should be adding more experimental 